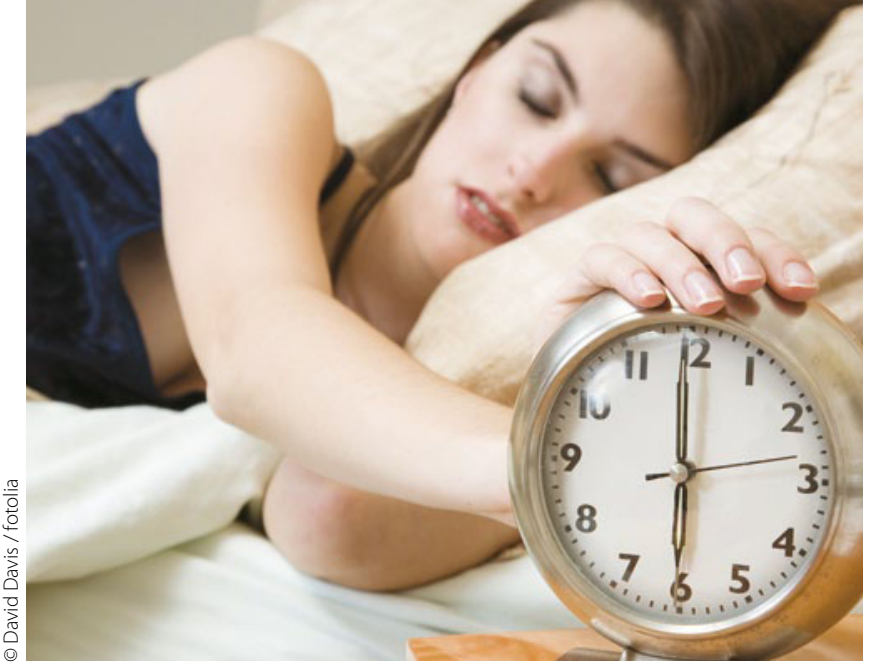

Frauen brauchen ca. sieben Stunden Schlaf, um geistig maximal leistungsfähig zu bleiben.

\title{
Zu viel oder zu wenig Schlaf beschleunigt das kognitive Altern
}

\author{
Der Schlaf beeinflusst die Hirnleistung. In einer Studie \\ wurde jetzt überprüft, welchen Einfluss die Schlafdauer \\ auf die kognitive Funktion hat.
}

- Wissenschaftler des University College London unterteilten in einer prospektiven Kohortenstudie 5431 freiwillige Probanden (1459 Frauen und 3972 Männer, 45-69 Jahre alt) in der sog. Whitehall-II-Studie hinsichtlich der Schlafdauer in fünf Gruppen (durchschnittlich $\leq 5,6,7,8, \geq 9$ Stunden pro Nacht). Die kognitiven Funktionen wurden über 5,4 Jahre mit sechs Tests gemessen.

Sowohl eine Abnahme (bei 25\% der Frauen und 18\% der Männer) als auch eine Zunahme der Schlafdauer (bei 7,4\% der Frauen und 8,6\% der Männer) wirkten sich auf die kognitive Leistung aus. Sie verschlechterte sich bei fünf von sechs Tests bei längerem und bei drei von sechs Tests bei kürzerem Schlaf. Optimale Testergebnisse erzielten Frauen, die ca. sieben Stunden pro Nacht schliefen. Bei Männern lag die ideale Schlafdauer zwischen sechs und acht Stunden. Längerer und kürzerer Schlaf wirkten sich negativ auf die Testergebnisse aus.

\section{Kommentar}

Ein adäquater und guter Schlaf ist fundamental für das Funktionieren und das Wohlbefinden des Menschen. Die negativen Effekte von zu viel oder zu wenig Schlaf auf die Gesundheit des Menschen erfahren durch die Wissenschaft immer mehr Aufmerksamkeit.

K. MALBERG =

\footnotetext{
- J. E. Ferrie et al.

(University College London, Department of Epidemiology and Public Health, Gower Street Campus, 1-19 Torrington Place, London WC1E 6BT, London, UK; e-mail: j.ferrie@ucl.ac.uk) Sleep duration and cognitive function. Change in sleep duration and cognitive function: findings from the Whitehall II study. Sleep 34 (2011) 565-573
} 